\title{
Large deviations for a stochastic Cahn-Hilliard equation in Hölder norm
}

\author{
L. Boulanba * $\quad$ M. Mellouk ${ }^{\dagger}$
}

\begin{abstract}
We consider a stochastic Cahn-Hilliard partial differential equation driven by a space-time white noise. We prove the Large Deviations Principle (LDP) for the law of the solutions in the Hölder norm. We use the weak convergence approach that reduces the proof to establishing basic qualitative properties for controlled analogues of the original stochastic system..
\end{abstract}

Keywords: Stochastic Cahn-Hilliard equation; Space-time white noise; Stochastic partial differential equations; Large deviations principle; Weak convergence method; Green function.

AMS Subject Classification: 60F10, 60H15, 60G15.

\section{Introduction}

In this paper we consider the following Stochastic Cahn-Hilliard equation with multiplicative space-time white noise, indexed by $\varepsilon>0$, given by

$$
\left\{\begin{array}{l}
\frac{\partial u^{\varepsilon}}{\partial t}(t, x)=-\Delta\left(\Delta u^{\varepsilon}(t, x)-f\left(u^{\varepsilon}(t, x)\right)\right)+\sqrt{\varepsilon} \sigma\left(u^{\varepsilon}(t, x)\right) \dot{W}(t, x), \quad(t, x) \in[0, T] \times \mathcal{D}, \\
u^{\varepsilon}(0, x)=u_{0}(x), \\
\frac{\partial u^{\varepsilon}}{\partial \nu}(t, x)=\frac{\partial \Delta u^{\varepsilon}}{\partial \nu}(t, x)=0, \text { on }[0, T] \times \partial \mathcal{D},
\end{array}\right.
$$

where $T>0, \mathcal{D}=[0, \pi]^{d}$ with $d=1,2,3, f$ is a polynomial of degree 3 with positive dominant coefficient such as $f=F^{\prime}$, where $F(u)=\left(1-u^{2}\right)^{2}$ is a double equal-well potential. The noise diffusion coefficient $\sigma$ is a bounded and Lipschitzian function, $W$ is a space-time Brownian sheet defined on some filtered probability space $\left(\Omega, \mathcal{F}, \mathcal{F}_{t}, P\right)$, and $\nu$ is the outward normal vector. The initial condition $u_{0}$ is a real-valued function satisfying some assumptions that will be specified later.

The deterministic Cahn-Hilliard equation (i.e., $\sigma \equiv 0$ in (1.1)) was introduced by Cahn and Hilliad in 1958 as a mathematical model of spinodal decomposition for a binary allow in order to determine the comprising species concentrations when the separation phase take place, see [11]. In this model, the function $f$ is the derivative of the homogeneous free energy $F$ that was given in its original form by

$$
F(u)=-\frac{\theta_{c}}{2} u^{2}+\frac{\theta}{2}((1+u) \ln (1+u)-(1-u) \ln (1-u)), \quad-1<u<1,
$$

where $\theta_{c}$ and $\theta$ are respectively proportional to the critical and the quenching temperatures. One can see [17] where it is rigourously justified that $F$ can be replaced in some circumstances by a polynomial of even degree

\footnotetext{
*Regional Center of the Trades of Education and Training, Souss-Massa. e-mail: 1.boulanba@gmail.com

†Université Paris Descartes, e-mail:mohamed.mellouk@parisdescartes.fr
} 
with a strictly positive dominant coefficient. For more details on physical aspects of this equation one can see e.g. ([20], [26], [28]).

Over the past three decades, different questions and properties related to the equation (1.1) have been the subject of many works. Indeed, the existence of the solution and of its density was established by Cardon-Weber in [13], a support theorem was showed in [5] by Bo and its co-authors, a Freidin-Wentzell large deviations principle was obtained by Shi et al. in [32]. Recently, Antonopoulou et al. [1] have attempted to go beyond bounded coefficient noisy term in order to improve the results of [13]. Also, the Cahn-Hilliard equation driven by non Gaussian perturbations was studied in a multitude of setting, we can cite e.g. [4] and [25].

Inspired by the pioneering works [35] and [24] on large deviations for diffusion stochastic processes, growing interest has been paid to this topic during the last three decades. This was thanks to its various applications in many scientific areas. Also, its nonlinear character and its connection with several mathematic theories make it an active field of theoretical researches. And besides a considerable literature about the large deviations for stochastic differential equations (SDEs), this aspect has been investigated for the most popular stochastic partial differential equations (SPDEs) and we here cite e.g. [27] for the stochastic heat equation, [12] for the stochastic Burgers equation, [15] for the stochastic wave equation of degree two and [33] for a reaction diffusion equation with non- Gaussian perturbation. Note that in all these works authors used the classical approach of Freidlin and Wentzell that was developed essentially in [2], [30] and [3]. For a complete and deep exposition of the topic of large deviations theory we refer to [18].

Recently, the weak convergence approach introduced by Ellis and Dupuis in [21] and developed in [6], [9] and [10] have gave a new impetus to the study of large deviations both to investigate new random dynamic systems or to revisit and improve anterior results of the point of relaxing assumptions or simplifying the proof. And taking advantage of this approach, many works on various SPDEs has been appeared in last few years. See for a short list e.g. ([8], [19], [22], [31], [29], [34]). The present paper fits into this optic.

It is worth mentioning that the weak convergence approach consists to use a Laplace principle and some variational representations for exponential functionals of infinite dimensional Brownian motion. The proofs are based on showing qualitative properties for controlled versions of the origin processes. This fact unable one to avoid well known difficulties of the classical approach when one wants establish exponential estimates that use approximation and discretization procedure.

In this work we show a large deviations principle for the stochastic Cahn-Hilliard equation in the Höder norm. Thereby, we improve the result of [32] that was given in terms of the uniform convergence topology. Moreover, our proofs are technically less demanding.

The present paper is organized as follows. Coming section contains basic backgrounds of large deviations theory and well known results about the solution of the equation (1.1). Section 3 gives the general framework of our work. In the last section, we announce and prove our main result.

\section{Preliminaries and main assumptions}

In this section we present some assumptions, preliminaries and standard definitions which are needed for the formulation of the problem.

\subsection{Large deviations}

For a family of random variables $\left\{X^{\varepsilon} ; \varepsilon>0\right\}$ defined on a probability space $(\Omega, \mathcal{F}, P)$ and taking values in a Polish space $\mathcal{E}$, the LDP is concerned with events $A$ for which probabilities $P\left(X^{\varepsilon} \in A\right)$ converges to zero exponentially fast as $\varepsilon \rightarrow 0$. The exponential decay rate of such probabilities are typically expressed in terms of a rate function $I$ mapping $\mathcal{E}$ into $[0, \infty]$.

Definition 2.1 The family of random variables $\left\{X^{\varepsilon} ; \varepsilon>0\right\}$ is said to satisfy the LDP with the good rate function (or action functional) $I: \mathcal{E} \rightarrow[0, \infty]$, on $\mathcal{E}$, if 
1. For each $M<\infty$ the level set $\{x \in \mathcal{E} ; I(x) \leq M\}$ is a compact subset of $\mathcal{E}$.

2. Large deviation upper bound: for any closed subset $F$ of $\mathcal{E}$

$$
\limsup _{\varepsilon \rightarrow 0^{+}} \varepsilon \log P\left(X^{\varepsilon} \in F\right) \leq-I(F) .
$$

3. Large deviation lower bound: for any open subset $O$ of $\mathcal{E}$

$$
\liminf _{\varepsilon \rightarrow 0^{+}} \varepsilon \log P\left(X^{\varepsilon} \in O\right) \geq-I(O) .
$$

Where, for $A \subset \mathcal{E}$, we define $I(A)=\inf _{x \in A} I(x)$.

The Freidlin-Wentzell theory [23] describes the path asymptotics, as $\varepsilon \rightarrow 0$, of probabilities of the large deviations of the solutions of small noise finite dimensional SDEs, away from its law if large number limite. For the case where the driving brownian motion is infinite dimensional, that covers the SPDEs, Budhiraja et al. [10] use certain variational representations to give a framework for proving large deviations for a variety of infinite dimensional systems.

In a many problems one is interested in obtaining exponential estimates on functions which are more general than indicator functions of closed or open sets. This leads to the study of the, so called, Laplace principle.

Definition 2.2 (Laplace principle) The family of random variables $\left\{X^{\varepsilon} ; \varepsilon>0\right\}$ defined on the Polish space $\mathcal{E}$, is said to satisfy the Laplace principle with rate function I if for any bounded continuous function $h: \mathcal{E} \rightarrow \mathbb{R}$,

$$
\lim _{\varepsilon \rightarrow 0} \varepsilon \log \mathbf{E}\left(\exp \left[-\frac{1}{\varepsilon} h\left(X^{\varepsilon}\right)\right]\right)=-\inf _{f \in \mathcal{E}}\{h(f)+I(f)\}
$$

where $\mathbf{E}$ is the expectation with respect to $P$.

In [35] and [7], Varadhan and Bryc established an equivalence between LDP and Laplace principle (LP) on a Polish space. In a view of this equivalence, the rest of this paper will be concerned with the study of the Laplace principle.

\subsection{Assumptions and mild solution}

Letting $q \geq 1$, we define $\|\cdot\|_{q}$ as the usual norm in $L^{q}(\mathcal{D})$. Assume that:

(H1) $f$ is a polynomial function of degree 3 with positive dominant coefficient.

(H2) $\sigma:$ is a bounded and Lipschitz function.

$(\mathrm{H} 3) u_{0} \in L^{p}(\mathcal{D})$ (for some $p \geq 4$ ) is continuous on $\mathcal{D}$.

(H3') $u_{0}$ is an $\gamma$-Hölder continuous function on $\left.\left.\mathcal{D}, \gamma \in\right] 0,1\right]$.

Following the J. B. Walsh approach [36], a rigorous meaning for solution of the equation (1.1) can be given by means of the following definition. 
Definition 2.3 (Mild solution) A jointly measurable and adapted process $\{u(t, x) ;(t, x) \in[0 . T] \times \mathcal{D}\}$ is called a mild solution of (1.1) with initial condition $u_{0}$ if it satisfies, for each $t \geq 0$ and a.s. for almost all $x \in \mathcal{D}$ the following evolution equation:

$$
\begin{aligned}
u^{\varepsilon}(t, x)= & \int_{\mathcal{D}} G_{t}(x, y) u_{0}(y) d y+\sqrt{\varepsilon} \int_{0}^{t} \int_{\mathcal{D}} G_{t-s}(x, y) \sigma\left(u^{\varepsilon}(s, y)\right) W(d s, d y) \\
& +\int_{0}^{t} \int_{\mathcal{D}} \Delta G_{t-s}(x, y) f\left(u^{\varepsilon}(s, y)\right) d s d y
\end{aligned}
$$

where $G_{t}(\cdot, \cdot)$ denotes the Green kernel corresponding to the operator $\frac{\partial}{\partial t}+\Delta^{2}$ with the Neumann boundary conditions. Note that some useful estimates concerning $G_{t}(\cdot, \cdot)$ are given in [13].

The following result of C. Cadon-Weber ([13], Theorem 1.3) asserts the existence and uniqueness of a solution to $(1.1)$.

Theorem 2.1 (Existence, uniqueness and the regularity of solutions) Under the assumptions (H1)-(H3), there exists a unique solution (in the Walsh's sense) of the equation (1.1) which satisfies

$$
\sup _{0 \leq t \leq T}\left(\mathbf{E}\|u(t, \cdot)\|_{p}^{q}\right)^{1 / q}<\infty
$$

for $q \geq p$ if $d \in\{1,2\}$ and for $p \leq q<\frac{6 p}{(6-p)^{+}}$if $d=3$. Moreover, under (H1)- (H3'), ([13], Theorem 4.1) gives the a.s. $\beta$-Hölder continuous property for the trajectories of the solution with $\beta \leq \frac{\gamma}{4}$ and $\beta<\frac{1}{2}\left(1-\frac{d}{4}\right)$.

Theorem 2.2 (The solution mapping of equation (1.1)). Assuming (H1)-(H3'). Let $\alpha \leq \frac{\gamma}{4} \wedge \frac{1}{2}\left(1-\frac{d}{4}\right)$ and set $\mathcal{E}_{0}=L^{p}(\mathcal{D}) \cap \mathcal{C}^{\gamma}(\mathcal{D})$, for some $\left.\left.p \geq 4, \gamma \in\right] 0,1\right]$. There exists a measurable function

$$
\mathcal{G}^{\varepsilon}: \mathcal{E}_{0} \times \mathcal{C}([0, T] \times \mathcal{D}: \mathbb{R}) \rightarrow C^{\alpha}\left([0, T], L^{p}(\mathcal{D})\right)
$$

such that, for any filtered probability space $\left(\Omega, \mathcal{F}, \mathcal{F}_{t}, P\right)$ with a Brownian sheet $W$ as above and $u_{0} \in \mathcal{E}_{0}$, $u^{\varepsilon}=\mathcal{G}^{\varepsilon}\left(u_{0}, \sqrt{\varepsilon} W\right)$, is the unique mild solution of (1.1) (with initial condition $u_{0}$ ) and satisfies (2.3).

In order to be able to apply the weak convergence approach for large deviations theory, we need a Polish space setting carrying the probability laws of the family $\left\{u^{\varepsilon}(t, x) ; \varepsilon \in(0,1],(t, x) \in[0, T] \times \mathcal{D}\right\}$. And regarding the Hölder property of $u$ we introduce the space $C^{\alpha}\left([0, T], L^{p}(\mathcal{D})\right)$ endowed with the following norm

$$
\|u\|_{\alpha, p}=\sup _{t \in[0, T]}\|u(t, \cdot)\|_{p}+\sup _{t \neq t^{\prime} t, t^{\prime} \in[0, T]} \frac{\left\|u(t, \cdot)-u\left(t^{\prime}, \cdot\right)\right\|_{p}}{\left|t-t^{\prime}\right|^{\alpha}},
$$

for $p \geq 4$ and $\alpha \in] 0,1\left[\right.$. And because our setting requires a Polish space state, we recall that if $\alpha^{\prime}<\alpha$, then the separable space $C^{\alpha^{\prime}, 0}\left([0, T], L^{p}(\mathcal{D})\right)$ of functions belonging to $C^{\alpha^{\prime}}\left([0, T], L^{p}(\mathcal{D})\right)$ and satisfying

$$
\lim _{\delta \rightarrow 0} \sup _{0<\left|t-t^{\prime}\right|<\delta, t \neq t^{\prime}} \frac{\left\|u(t, \cdot)-u\left(t^{\prime}, \cdot\right)\right\|_{p}}{\left|t-t^{\prime}\right| \alpha^{\prime}}=0
$$

is a polish space containing $C^{\alpha}\left([0, T], L^{p}(\mathcal{D})\right)$. Hence, we can restrict ourselves in all the sequel to the space $\mathcal{E}^{\alpha}:=C^{\alpha, 0}\left([0, T], L^{p}(\mathcal{D})\right)$ for $\alpha<\frac{\gamma}{4} \wedge \frac{1}{2}\left(1-\frac{d}{4}\right)$.

\section{Framework for the Laplace Principle}

Here, we first review an important result given by Budhiraja et al. [10], and which ensures the obtention of Laplace principle. 


\subsection{Laplace principle of functionals of Brownian sheet.}

Consider the filtered probability space $\left(\Omega, \mathcal{F}, \mathcal{F}_{t}, P\right)$ defined in the introduction, and let $\mathcal{E}_{0}$ and $\mathcal{E}$ be Polish spaces such that the initial condition $u_{0}$ takes values in a compact subspace of $\mathcal{E}_{0}$.

Moreover, let $\left\{\mathcal{G}^{\varepsilon}: \mathcal{E}_{0} \times \mathcal{C}([0, T] \times \mathcal{D} ; \mathbb{R}) \rightarrow \mathcal{E}, \varepsilon>0\right\}$ be a family of measurable maps.

For $u_{0} \in \mathcal{E}_{0}$, Define

$$
X^{\varepsilon, u_{0}}:=\mathcal{G}^{\varepsilon}\left(u_{0}, \sqrt{\varepsilon} W\right) .
$$

In the sequel we will give sufficient conditions for the Laplace principle for $X^{\varepsilon, u_{0}}$ to hold uniformly in $u_{0}$ for compact subsets of $\mathcal{E}_{0}$.

For $N \in \mathbb{N}$, consider the following

$$
S^{N}=\left\{\phi \in L^{2}([0, T] \times \mathcal{D}): \int_{0}^{T} \int_{\mathcal{D}} \phi^{2}(s, y) d s d y \leq N\right\}
$$

which is a compact metric space, equipped with the weak topology on $L^{2}([0, T] \times \mathcal{D})$.

Let $\mathcal{P}_{2}$ be the class of all predictable processes $\phi$ such that $\int_{0}^{T} \int_{\mathcal{D}} \phi^{2}(s, y) d s d y<\infty$, a.s. Also, define

$$
\mathcal{P}_{2}^{N}=\left\{v(\omega) \in \mathcal{P}_{2}: v(\omega) \in S^{N}, P-a . s\right\},
$$

the space of controls.

The following condition is the standing assumption of Theorem 3.1 which states the Laplace principle for the family $\left\{X^{\varepsilon, u_{0}}\right\}_{\varepsilon>0}$ defined by (3.5). For $u \in L^{2}([0, T] \times \mathcal{D})$, define $\mathcal{I}(u) \in \mathcal{C}([0, T] \times \mathcal{D}: \mathbb{R})$ as

$$
\mathcal{I}(u)(t, x):=\int_{0}^{t} \int_{0}^{x} u(s, y) d s d y .
$$

Assumption $(\mathcal{A})$ : There exists a measurable map $\mathcal{G}^{0}: \mathcal{E}_{0} \times \mathcal{C}([0, T] \times \mathcal{D} ; \mathbb{R}) \rightarrow \mathcal{E}$ such that the following hold:

(A1) For every $M<\infty$ and a compact set $K \subset \mathcal{E}_{0}$, the set

$$
\Gamma_{M, K}:=\left\{\mathcal{G}^{0}\left(u_{0}, \mathcal{I}(u)\right) ; u \in S^{M}, u_{0} \in K\right\}
$$

is a compact subset of $\mathcal{E}$.

(A2) Consider $M<\infty$ and a family $\left\{v^{\varepsilon}: \varepsilon>0\right\} \subset \mathcal{P}_{2}{ }^{M}$, and $\left\{u_{0}^{\varepsilon}\right\} \subset \mathcal{E}_{0}$ such that $v^{\varepsilon} \rightarrow v$ and $u_{0}^{\varepsilon} \rightarrow u_{0}$ in distribution (as $S^{N}$-valued random elements) as $\varepsilon \rightarrow 0$. Then

$$
\mathcal{G}^{\varepsilon}\left(u_{0}^{\varepsilon}, \sqrt{\varepsilon} W+\mathcal{I}\left(v^{\varepsilon}\right)\right) \rightarrow \mathcal{G}^{0}\left(u_{0}, \mathcal{I}(u)\right),
$$

in distribution as $\varepsilon \rightarrow 0$.

For $h \in \mathcal{E}$, and $u_{0} \in \mathcal{E}_{0}$, define the rate function

$$
I_{u_{0}}(h):=\inf _{\left\{v \in L^{2}([0, T] \times \mathcal{D}): h=\mathcal{G}^{0}\left(u_{0}, \mathcal{I}(v)\right)\right\}}\left\{\frac{1}{2} \int_{0}^{T} \int_{\mathcal{D}} v^{2}(s, y) d y d s\right\},
$$

where the infimum over an empty set is taken to be $\infty$.

The following theorem is due to Budhiraja et al.( [10], Theorem 7) and states the Laplace principle for the family $X^{\varepsilon, u_{0}}$. 
Theorem 3.1 (Theorem 7 in [10]) Let $\mathcal{G}^{0}: \mathcal{E}_{0} \times \mathcal{C}([0, T] \times \mathcal{D} ; \mathbb{R}) \rightarrow \mathcal{E}$ be a measurable map satisfying assumption $(\mathcal{A})$. Suppose that for all $h \in \mathcal{E}, u_{0} \rightarrow I_{u_{0}}(h)$ is a lower semi-continuous map from $\mathcal{E}_{0}$ to $[0, \infty]$. Then for every $u_{0} \in \mathcal{E}_{0}, I_{u_{0}}(h): \mathcal{E} \rightarrow[0, \infty]$, is a rate function on $\mathcal{E}$ and the family $\left\{I_{u_{0}}, u_{0} \in \mathcal{E}\right\}$ of rate functions has compact level sets on compacts. Furthermore, the family $X^{\varepsilon, u_{0}}$ satisfies the Laplace principle on $\mathcal{E}$ with rate function $I_{u_{0}}$ defined by (3.6), uniformly in $u_{0}$ on compact subsets of $\mathcal{E}_{0}$.

\subsection{The controlled and limiting equations for the spde (1.1)}

In the context of the spde under our study, $\mathcal{E}_{0}=L^{p}(\mathcal{D}) \cap \mathcal{C}^{\gamma}(\mathcal{D})$, for some $\left.\left.p \geq 4, \gamma \in\right] 0,1\right]$ is the space of the initial condition, and $\mathcal{E}=\mathcal{E}^{\alpha}:=C^{\alpha, 0}\left([0, T], L^{p}(\mathcal{D})\right)$ for $\alpha<\frac{\gamma}{4} \wedge \frac{1}{2}\left(1-\frac{d}{4}\right)$ the space of solutions.

The solution map of equation (1.1) is $u^{\varepsilon}=\mathcal{G}^{\varepsilon}\left(u_{0}, \sqrt{\varepsilon} W\right)$. Then, for $v \in \mathcal{P}_{2}^{N}, u^{\varepsilon, v}:=\mathcal{G}^{\varepsilon}\left(u_{0}, \sqrt{\varepsilon} W+\mathcal{I}(v)\right)$ is the solution map of the stochastic controlled equation for the spde (1.1):

$$
\frac{\partial u^{\varepsilon, v}}{\partial t}(t, x)=-\Delta\left(\Delta u^{\varepsilon, v}(t, x)-f\left(u^{\varepsilon, v}(t, x)\right)\right)+\sqrt{\varepsilon} \sigma\left(u^{\varepsilon, v}(t, x)\right) \frac{\partial^{2} W}{\partial t \partial x}+\sigma\left(u^{\varepsilon, v}(t, x)\right) v(t, x),
$$

whose mild solution is

$$
\begin{aligned}
u^{\varepsilon, v}(t, x)= & \int_{\mathcal{D}} G_{t}(x, y) u_{0}(y) d y+\sqrt{\varepsilon} \int_{0}^{t} \int_{\mathcal{D}} G_{t-s}(x, y) \sigma\left(u^{\varepsilon, v}(s, y)\right) W(d s, d y) \\
& +\int_{0}^{t} \int_{\mathcal{D}} \Delta G_{t-s}(x, y) f\left(u^{\varepsilon, v}(s, y)\right) d s d y+\int_{0}^{t} \int_{\mathcal{D}} G_{t-s}(x, y) \sigma\left(u^{\varepsilon, v}(s, y)\right) v(s, y) d s d y .
\end{aligned}
$$

Also, define the map $\mathcal{G}^{0}\left(u_{0}, \mathcal{I}(v)\right):=u^{v}$, where $u^{v}$ is the solution of the following zero-noise equation:

$$
\begin{aligned}
u^{v}(t, x)= & \int_{\mathcal{D}} G_{t}(x, y) u_{0}(y) d y+\int_{0}^{t} \int_{\mathcal{D}} G_{t-s}(x, y) \sigma\left(u^{v}(s, y)\right) v(s, y) d s d y \\
& +\int_{0}^{t} \int_{\mathcal{D}} \Delta G_{t-s}(x, y) f\left(u^{v}(s, y)\right) d s d y .
\end{aligned}
$$

The following theorem gives a statement of existence and uniqueness for the solution of the stochastic controlled equation given by (3.7).

Theorem 3.2 (Existence and uniqueness of controlled process) Assuming $(H 1)-(H 3)$. Let $\mathcal{G}^{\varepsilon}$ denote the solution mapping, and let $v \in \mathcal{P}_{2}^{N}$ for some $N \in \mathbb{N}$. Define

$$
u^{\varepsilon, v}=\mathcal{G}^{\varepsilon}\left(u_{0}, \sqrt{\varepsilon} W+\mathcal{I}(v)\right),
$$

then $u^{\varepsilon, v}$ is the unique solution of equation (3.7), which satisfies

$$
\sup _{\varepsilon \leq 1} \sup _{v \in \mathcal{P}_{2}^{N}} \sup _{0 \leq t \leq T} \mathbf{E}\left(\left\|u^{\varepsilon, v}(t, \cdot)\right\|_{p}^{q}\right)<\infty
$$

for $q \geq p$ if $d=1,2$, and $p \leq q<\frac{6 p}{(6-p)^{+}}$in the case $d=3$.

Proof. For $v \in \mathcal{P}_{2}^{N}$, set

$$
d Q^{\varepsilon, v}:=\exp \left\{-\frac{1}{\sqrt{\varepsilon}} \int_{0}^{t} \int_{D} v(s, y) W(d s, d y)-\frac{1}{2 \varepsilon} \int_{0}^{t} \int_{D} v(s, y)^{2} d s d y\right\} d P .
$$

Since is defined by an exponential martingale, $Q^{\varepsilon, v}$ is a probability measure on $\Omega$. And by Girsanov theorem the process

$$
\tilde{W}(d t, d x)=W(d t, d x)+\frac{1}{\sqrt{\varepsilon}} \int_{0}^{t} \int_{D} v(s, y) d s d y
$$


is a space-time white noise on the space $\Omega$ under the probability measure $Q^{\varepsilon, v}$. Rewriting (3.7) using $\tilde{W}(d t, d x)$ we obtain (2.2) with $\tilde{W}(d t, d x)$ in place of $W(d t, d x)$. Let $\mathbf{u}$ be the unique solution of $(2.2)$ with $\tilde{W}(d t, d x)$ on the space $\left(\Omega, \mathcal{F}, Q^{\varepsilon, v}\right)$. Then $\mathbf{u}$ satisfies $(3.7), Q^{\varepsilon, v}$ a.s. And by equivalence of probabilities, then $\mathbf{u}$ satisfies (3.7), $P$ a.s.

For the uniqueness, if $\mathbf{u}_{1}$ and $\mathbf{u}_{2}$ are two solutions of (3.7) on $(\Omega, \mathcal{F}, P)$, then $\mathbf{u}_{1}$ and $\mathbf{u}_{2}$ are solutions of (2.2) governed by $\tilde{W}(d t, d x)$ on $\left(\Omega, \mathcal{F}, Q^{\varepsilon, v}\right)$. By the uniqueness of the solution of (2.2), we obtain $\mathbf{u}_{1}=\mathbf{u}_{2}, Q^{\varepsilon, v}$ a.s. And by equivalence of probabilities, we obtain $\mathbf{u}_{1}=\mathbf{u}_{2}, P$ a.s.

Concerning the estimate (3.9), it holds true for the three first terms by the estimations $(2.16),(2.17)$ and $(2.35)$ in [13]. It remains to show it for the last term. Indeed, by the estimation (1.11) in [13], there exists a constant $c>0$ such that

$$
\left\|\int_{0}^{t} \int_{D} G_{t-s}(\cdot, y) \sigma\left(u^{\varepsilon, v}(s, y)\right) v(s, y) d s d y\right\|_{p} \leq c \int_{0}^{t}(t-s)^{\frac{d}{4}\left(\frac{1}{r}-1\right)}\left\|\sigma\left(u^{\varepsilon, v}(s, \cdot)\right) v(s, \cdot)\right\|_{\rho} d s,
$$

where $\rho \in[1, p]$ and $\frac{1}{r}=\frac{1}{p}-\frac{1}{\rho}+1$. Using the boundedness of $\sigma$, taking $\rho=2$ in the last inequality and applying Cauchy Schwarz inequality we get a.s.

$$
\begin{aligned}
\left\|\int_{0}^{t} \int_{D} G_{t-s}(\cdot, y) \sigma\left(u^{\varepsilon, v}(s, y)\right) v(s, y) d s d y\right\|_{p} & \leq \frac{c}{\frac{d}{2}\left(\frac{1}{r}-1\right)+1} T^{\frac{d}{2}\left(\frac{1}{r}-1\right)+1}\|v\|_{\mathcal{H}_{T}} \\
& \leq \frac{c}{\frac{d}{2}\left(\frac{1}{r}-1\right)+1} T^{\frac{d}{2}\left(\frac{1}{r}-1\right)+1} N .
\end{aligned}
$$

Note that, with the condition $p \geq 4$, there exists $r$ satisfying (3.10) that can be taken in $\left[\frac{4}{3}, 3[\right.$. Then

$$
\mathbf{E}\left(\left\|\int_{0}^{t} \int_{D} G_{t-s}(\cdot, y) \sigma\left(u^{\varepsilon, v}(s, y)\right) v(s, y) d s d y\right\|_{p}^{q}\right)<\infty .
$$

Hence, (3.9) holds.

Remark 3.1 (Hölder regularity of controlled and limiting processes) Assuming $(H 1)-\left(H 3^{\prime}\right)$. Both processes $\left\{u^{\varepsilon, v}(t, \cdot) ; t \in[0, T]\right\}$ and $\left\{u^{v}(t, \cdot) ; t \in[0, T]\right\}$, defined by (3.7) and (3.8) respectively, live in the space $\mathcal{E}^{\alpha}$.

Proof.

The Hölder regularity for these two processes can be obtained by arguing as in the point ii) of the proof of Theorem 4.1.

For $h \in \mathcal{E}^{\alpha}$, and $u_{0} \in \mathcal{E}_{0}$, define the rate function

$$
I_{u_{0}}(h):=\inf _{v}\left\{\frac{1}{2} \int_{0}^{T} \int_{\mathcal{D}} v^{2}(s, y) d y d s\right\},
$$

where the infimum is taken over all $v \in L^{2}([0, T] \times \mathcal{D})$ such that

$$
\begin{aligned}
h(t, x) & =\int_{\mathcal{D}} G_{t}(x, y) u_{0}(y) d y+\int_{0}^{t} \int_{\mathcal{D}} G_{t-s}(x, y) \sigma(h(s, y)) v(s, y) d s d y \\
& +\int_{0}^{t} \int_{\mathcal{D}} \Delta G_{t-s}(x, y) f(h(s, y)) d s d y .
\end{aligned}
$$

Note that under assumptions (H1)-(H3), for every $v \in \mathcal{P}_{2}^{N}$, the equation (3.13) admits a unique solution which belongs to $C\left([0, T], L^{p}(D)\right)$, and moreover

$$
\sup _{t \in[0, T]}\left\|u^{v}(t, \cdot)\right\|_{p}^{q}<\infty
$$

for $q \geq p$ if $d \in\{1,2\}$ and for $p \leq q \leq \frac{6 p}{(6-p)^{+}}$if $d=3$. The proof is omitted since is similar to that in Theorem 3.1 of [13] but by replacing the stochastic integral by the integral containing $v$. 


\section{The main result}

The main result of this paper is the following:

Theorem 4.1 Under the assumptions (H1)-(H3'), the law of the solution $\left\{u^{\varepsilon} ; \varepsilon \in(0,1]\right\}$, defined by (2.2), satisfies, on $\mathcal{E}^{\alpha}$, a large deviation principle with the rate function $I_{u_{0}}$, defined by (3.12), uniformly for $u_{0}$ in compact subsets of $\mathcal{E}_{0}$.

In view of Theorem 3.1, to prove Theorem 4.1 it suffices to verify conditions (A1) and (A2).

Remark 4.1 This result improve that of Shi and al. [32] where the LDP was established by the classical approach in the space $C\left([0, T] ; L^{p}(\mathcal{D})\right)$ equipped with the topology of uniform convergence.

Proof of Theorem 4.1 As mentioned above, here we will show that the conditions $(A 1)$ and $(A 2)$ hold. In a first time we deal with $(A 2)$. That is, we need to show that for all $q \geq p$ we have

$$
\left\|u^{\varepsilon, v^{\varepsilon}}(t)-u^{v}(t)\right\|_{\alpha, p}^{q} \longrightarrow 0 \text { in probability as } \varepsilon \longrightarrow 0 \text {. }
$$

To do it, we will use a localization argument introduced in [14]. For $0 \leq t \leq T, \varepsilon \in] 0,1]$ and $M>0$, define the following event

$$
A_{\varepsilon}^{M}(t)=\left\{w \in \Omega ; \sup _{s \in[0, t]}\left\|u^{\varepsilon, v^{\varepsilon}}(s)\right\|_{p} \vee \sup _{s \in[0, t]}\left\|u^{v}(s)\right\|_{p} \leq M\right\}
$$

Notice that $A_{\varepsilon}^{M}(t) \in \mathcal{F}_{t}$, and set

$$
Y_{\varepsilon}(t):=u^{\varepsilon, v^{\varepsilon}}(t)-u^{v}(t)
$$

Owing to (3.9) and (3.14), we have $P\left(A_{\varepsilon}^{M}(T)^{c}\right) \longrightarrow 0$ as $\varepsilon \longrightarrow 0$ and $M \longrightarrow \infty$. Then, by using Lemma A.1 in [14], it suffices to show

i) for all $t \in[0, T]$;

$$
\lim _{\varepsilon \longrightarrow 0} \mathbf{E}\left[\mathbf{1}_{A_{\varepsilon}^{M}(t)}\left\|Y_{\varepsilon}(t)\right\|_{p}^{q}\right]=0
$$

ii) there exists $\beta>0$ such that for all $t, t^{\prime} \in[0, T]$,

$$
\sup _{\varepsilon \in] 0,1[} \mathbf{E}\left[\mathbf{1}_{A_{\varepsilon}^{M}(T)}\left\|Y_{\varepsilon}(t)-Y_{\varepsilon}\left(t^{\prime}\right)\right\|_{p}^{q}\right] \leq c\left|t-t^{\prime}\right|^{\beta q}
$$

To prove 4.16 , we write

$$
\begin{aligned}
Y_{\varepsilon}(t)= & \sqrt{\varepsilon} \int_{0}^{t} \int_{D} G_{t-s}(\cdot, y) \sigma\left(u^{\varepsilon, v^{\varepsilon}}(s, y)\right) W(d s, d y)+\int_{0}^{t} \int_{D} \Delta G_{t-s}(\cdot, y)\left[f\left(u^{\varepsilon, v^{\varepsilon}}(s, y)\right)-f\left(u^{v}(s, y)\right)\right] d s d y \\
& +\int_{0}^{t} \int_{D} G_{t-s}(\cdot, y) \sigma\left(u^{\varepsilon, v^{\varepsilon}}(s, y)\right)\left[v^{\varepsilon}(s, y)-v(s, y)\right] d s d y \\
& +\int_{0}^{t} \int_{D} G_{t-s}(\cdot, y)\left[\sigma\left(u^{\varepsilon, v^{\varepsilon}}(s, y)\right)-\sigma\left(u^{v}(s, y)\right)\right] v(s, y) d s d y \\
& \equiv \sum_{i=1}^{4} J_{i}^{\varepsilon}(t) .
\end{aligned}
$$

Then

$$
\begin{aligned}
\mathbf{E}\left[\mathbf{1}_{A_{\varepsilon}^{M}(t)}\left\|Y_{\varepsilon}(t)\right\|_{p}^{q}\right] & \leq c \sum_{i=1}^{4} \mathbf{E}\left(\mathbf{1}_{A_{\varepsilon}^{M}(t)}\left\|J_{i}(t)\right\|_{p}^{q}\right) \\
& \leq c \sum_{i=1, i \neq 2}^{4} \mathbf{E}\left(\left\|J_{i}(t)\right\|_{p}^{q}\right)+c \mathbf{E}\left(\mathbf{1}_{A_{\varepsilon}^{M}(t)}\left\|J_{2}(t)\right\|_{p}^{q}\right)
\end{aligned}
$$


For $J_{1}^{\varepsilon}(t)$, first we apply Hölder inequality and we get

$$
\mathbf{E}\left(\left\|J_{1}^{\varepsilon}(t)\right\|_{p}^{q}\right)=\mathbf{E}\left(\int_{D}\left|J_{1}^{\varepsilon}(t, x)\right|^{p} d x\right)^{\frac{q}{p}} \leq c \int_{D} \mathbf{E}\left|J_{1}^{\varepsilon}(t, x)\right|^{q} d x .
$$

Later we use Burkholder inequality, the boundedness of $\sigma$ and the estimation (5.33)

$$
\begin{aligned}
\mathbf{E}\left|J_{1}^{\varepsilon}(t, x)\right|^{q} & \leq c \varepsilon^{\frac{q}{2}}\left(\int_{0}^{t} \int_{D} G_{t-s}^{2}(x, y) \sigma^{2}\left(u^{\varepsilon, v^{\varepsilon}}(s, y)\right) d s d y\right)^{q / 2} \\
& \leq c \varepsilon^{\frac{q}{2}}\left(\int_{0}^{t} \int_{D} G_{t-s}^{2}(x, y) d s d y\right)^{q / 2} \\
& <c \varepsilon^{\frac{q}{2}}
\end{aligned}
$$

Concerning $J_{2}^{\varepsilon}(t)$, using (3.16) in [4] and Hölder inequality we get for $1 \leq \rho \leq p$ and $1<\gamma \leq q$

$$
\begin{aligned}
\mathbf{E}\left(\mathbf{1}_{A_{\varepsilon}^{M}(t)}\left\|J_{2}^{\varepsilon}(t)\right\|_{p}^{q}\right) & \leq c \mathbf{E}\left(\mathbf{1}_{A_{\varepsilon}^{M}(t)} \int_{0}^{t}\left\|f\left(u^{\varepsilon, v^{\varepsilon}}(s, \cdot)\right)-f\left(u^{v}(s, \cdot)\right)\right\|_{\rho}^{\gamma} d s\right)^{\frac{q}{\gamma}} \\
& \leq c \mathbf{E}\left(\int_{0}^{t} \mathbf{1}_{A_{\varepsilon}^{M}(s)}\left\|f\left(u^{\varepsilon, v^{\varepsilon}}(s, \cdot)\right)-f\left(u^{v}(s, \cdot)\right)\right\|_{\rho}^{q} d s\right) .
\end{aligned}
$$

Note that, for the last inequality, taking into account the facts that $A_{\varepsilon}^{M}(t) \in \mathcal{F}_{t}$ and $A_{\varepsilon}^{M}(t) \subset A_{\varepsilon}^{M}(s)$ for $0 \leq s \leq t$, we have used the following upper estimate

$$
\left|\mathbf{1}_{A_{\varepsilon}^{M}(t)} \int_{0}^{t} \int_{D} \phi(s, y) d y d s\right| \leq\left|\int_{0}^{t} \int_{D} \mathbf{1}_{A_{\varepsilon}^{M}(s)} \phi(s, y) d y d s\right|,
$$

for a measurable function $\phi: \Omega \times[0, T] \times D \longrightarrow \mathbb{R}$. One can see Remark 3.2. in [14].

And, since $f$ is a polynomial function of degree 3 , we can write

$$
\begin{gathered}
\left\|f\left(u^{\varepsilon, v^{\varepsilon}}(s, \cdot)\right)-f\left(u^{v}(s, \cdot)\right)\right\|_{\rho}^{q} \leq c\left[\left\|u^{\varepsilon, v^{\varepsilon}}(s, \cdot)-u^{v}(s, \cdot)\right\|_{\rho}^{q}+\left\|u^{\varepsilon, v^{\varepsilon}}(s, \cdot)^{2}-u^{v}(s, \cdot)^{2}\right\|_{\rho}^{q}\right. \\
+\|\left(u^{\varepsilon, v^{\varepsilon}}(s, \cdot)^{3}-u^{v}(s, \cdot)^{3} \|_{\rho}^{q}\right] .
\end{gathered}
$$

Taking $\rho=\frac{p}{3}$, we have

$$
\begin{aligned}
& \left\|u^{\varepsilon, v^{\varepsilon}}(s, \cdot)-u^{v}(s, \cdot)\right\|_{\rho}^{q} \leq c\left\|u^{\varepsilon, v^{\varepsilon}}(s, \cdot)-u^{v}(s, \cdot)\right\|_{p}^{q} ; \\
& \left\|u^{\varepsilon, v^{\varepsilon}}(s, \cdot)^{2}-u^{v}(s, \cdot)^{2}\right\|_{\rho}^{q} \leq c\left\|u^{\varepsilon, v^{\varepsilon}}(s, \cdot)-u^{v}(s, \cdot)\right\|_{p}^{q}\left(\left\|u^{\varepsilon, v^{\varepsilon}}(s, \cdot)\right\|_{p}^{q}+\left\|u^{v}(s, \cdot)\right\|_{p}^{q}\right) ; \\
& \left\|u^{\varepsilon, v^{\varepsilon}}(s, \cdot)^{3}-u^{v}(s, \cdot)^{3}\right\|_{\rho}^{q} \leq c\left\|u^{\varepsilon, v^{\varepsilon}}(s, \cdot)-u^{v}(s, \cdot)\right\|_{p}^{q} \\
& \times\left(\left\|u^{\varepsilon, v^{\varepsilon}}(s, \cdot)\right\|_{p}^{q}+\left\|u^{\varepsilon, v^{\varepsilon}}(s, \cdot)\right\|_{p}^{q}\left\|u^{v}(s, \cdot)\right\|_{p}^{q}+\left\|u^{v}(s, \cdot)\right\|_{p}^{q}\right) .
\end{aligned}
$$

Then

$$
\mathbf{E}\left(\mathbf{1}_{A_{\varepsilon}^{M}(t)}\left\|J_{2}^{\varepsilon}(t)\right\|_{p}^{q}\right) \leq c \int_{0}^{T} \mathbf{E}\left(\mathbf{1}_{A_{\varepsilon}^{M}(s)}\left\|Y_{\varepsilon}(s)\right\|_{p}^{q}\right) d s .
$$


For $J_{3}^{\varepsilon}(t)$, Hölder inequality, the boundedness of $\sigma$ and the Cauchy schwarz inequality yield

$$
\begin{aligned}
\mathbf{E}\left(\left\|J_{3}^{\varepsilon}(t)\right\|_{p}^{q}\right) & \leq c \int_{D} \mathbf{E}\left|J_{3}(t, x)\right|^{q} d x \\
& \leq c \int_{D} \mathbf{E}\left(\int_{0}^{t} \int_{D} G_{t-s}(x, y)\left|v^{\varepsilon}(s, y)-v(s, y)\right| d s d y\right)^{q} d x \\
& \leq c \sup _{x \in D}\left(\int_{0}^{t} \int_{D} G_{t-s}^{2}(x, y) d y\right)^{q} \mathbf{E}\left(\left\|v^{\varepsilon}-v\right\|_{\mathcal{H}_{T}}^{q}\right) \\
& \leq c \mathbf{E}\left(\left\|v^{\varepsilon}-v\right\|_{\mathcal{H}_{T}}^{q}\right) .
\end{aligned}
$$

For $J_{4}^{\varepsilon}(t)$, the same arguments as before yield that a.s we have

$$
\begin{aligned}
\left\|J_{4}^{\varepsilon}(t)\right\|_{p}^{q} & \leq\|v\|_{\mathcal{H}_{T}}^{q}\left(\int_{D}\left(\int_{0}^{t} \int_{D} G_{t-s}^{2}(x, y)\left[\sigma\left(u^{\varepsilon, v^{\varepsilon}}(s, y)\right)-\sigma\left(u^{v}(s, y)\right]^{2} d s d y\right)^{\frac{p}{2}} d x\right)^{q / p}\right. \\
& \leq N^{q}\left(\int_{D}\left[\left(\int_{0}^{t} \int_{D} G_{s}^{2}(x, y) d s d y\right)^{\frac{p}{2}-1} \int_{0}^{t} \int_{D} G_{t-s}^{2}(x, y)\left|Y_{\varepsilon}(s, y)\right|^{p} d s d y\right] d x\right)^{q / p} \\
& \leq c\left(\int_{D}\left[\int_{0}^{t} \int_{D} G_{t-s}^{2}(x, y)\left|u^{\varepsilon, v^{\varepsilon}}(s, y)-u^{v}(s, y)\right|^{p} d s d y\right] d x\right)^{q / p} \\
& \leq c\left(\int_{0}^{t}\left(\int_{D} G_{t-s}^{2}(x, y) d x\right) \int_{D}\left|Y_{\varepsilon}(s, y)\right|^{p} d y d s\right)^{q / p} \\
& \leq c\left(\int_{0}^{t}(t-s)^{-\frac{d}{4}}\left\|Y_{\varepsilon}(s, y)\right\|_{p}^{p} d s\right)^{q / p} \\
& \leq c\left(\int_{0}^{t}(t-s)^{-\frac{d}{4}}\right)^{\frac{q}{p}-1} \int_{0}^{t}(t-s)^{-\frac{d}{4}}\left\|Y_{\varepsilon}(s, y)\right\|_{p}^{q} d s \\
& \leq c \int_{0}^{t}(t-s)^{-\frac{d}{4}}\left\|Y_{\varepsilon}(s)\right\|_{p}^{q} d s .
\end{aligned}
$$

Hence, combining (4.18)-(4.23) we obtain

$$
\mathbf{E}\left(\mathbf{1}_{A_{\varepsilon}^{M}(t)}\left\|Y_{\varepsilon}(t)\right\|_{p}^{q}\right) \leq c\left(\varepsilon^{\frac{q}{2}}+\mathbf{E}\left(\left\|v^{\varepsilon}-v\right\|_{\mathcal{H}_{T}}^{q}\right)+\int_{0}^{T}\left(1+(t-s)^{-\frac{d}{4}}\right) \mathbf{E}\left(\mathbf{1}_{A_{\varepsilon}^{M}(s)}\left\|Y_{\varepsilon}(s)\right\|_{p}^{q}\right) d s\right) .
$$

We obtain (4.16) by applying a version of Gronwall lemma given by (Lemma 15, [16]).

To prove (4.17), consider $t ; t^{\prime} \in[0, T]$ such that $t<t^{\prime}$. We have

$$
Y_{\varepsilon}(t)-Y_{\varepsilon}\left(t^{\prime}\right)=\left(u^{\varepsilon, v^{\varepsilon}}(t)-u^{\varepsilon, v^{\varepsilon}}\left(t^{\prime}\right)\right)-\left(u^{v}(t)-u^{v}\left(t^{\prime}\right)\right) .
$$

Then

$$
\mathbf{E}\left(\mathbf{1}_{A_{\epsilon}^{M}(T)}\left\|Y_{\varepsilon}(t)-Y_{\varepsilon}\left(t^{\prime}\right)\right\|_{p}^{q}\right) \leq \mathbf{E}\left(\mathbf{1}_{A_{\epsilon}^{M}(T)}\left\|u^{\varepsilon, v^{\varepsilon}}(t)-u^{\varepsilon, v^{\varepsilon}}\left(t^{\prime}\right)\right\|_{p}^{q}\right)+P\left(A_{\epsilon}^{M}(T)\right)\left\|u^{v}(t)-u^{v}\left(t^{\prime}\right)\right\|_{p}^{q} .
$$

At beginning we deal with the first term and we write (3.7) as

$$
u^{\varepsilon, v^{\varepsilon}}(t, \cdot):=I_{0}^{\varepsilon, v^{\varepsilon}}(t, \cdot)+I_{1}^{\varepsilon, v^{\varepsilon}}(t, \cdot)+I_{2}^{\varepsilon, v^{\varepsilon}}(t, \cdot)+I_{3}^{\varepsilon, v^{\varepsilon}}(t, \cdot),
$$


where $I_{i}^{\varepsilon, v^{\varepsilon}}(t, x)$ stands for the $i$-th term in (3.7). By Lemma 2.2. in [13], there exists a constant $c>0$ such that

$$
\left\|I_{0}^{\varepsilon, v^{\varepsilon}}(t)-I_{0}^{\varepsilon, v^{\varepsilon}}\left(t^{\prime}\right)\right\|_{p}^{q} \leq c\left|t-t^{\prime}\right|^{\frac{q \gamma}{4}} .
$$

The same reference gives the existence of $\beta>0$ such that $0<\beta<\frac{1}{2}\left(1-\frac{d}{4}\right)$ such that

$$
\mathbf{E}\left(\left\|I_{1}^{\varepsilon, v^{\varepsilon}}(t)-I_{1}^{\varepsilon, v^{\varepsilon}}\left(t^{\prime}\right)\right\|_{p}^{q}\right) \leq c \sqrt{\varepsilon}\left|t-t^{\prime}\right|^{\beta q}
$$

Concerning $I_{2}^{\varepsilon, v^{\varepsilon}}(t)$ we have

$$
\begin{aligned}
I_{2}^{\varepsilon, v^{\varepsilon}}\left(t^{\prime}\right)-I_{2}^{\varepsilon, v^{\varepsilon}}(t)= & \int_{0}^{t} \int_{D} \Delta\left[G_{t^{\prime}-s}(\cdot, y)-G_{t-s}(\cdot, y)\right] f\left(u^{\varepsilon, v^{\varepsilon}}(s, y)\right) d s d y \\
& +\int_{t}^{t^{\prime}} \int_{D} \Delta G_{t^{\prime}-s}(\cdot, y) f\left(u^{\varepsilon, v^{\varepsilon}}(s, y)\right) d s d y \\
\equiv & I_{2,1}^{\varepsilon, v^{\varepsilon}}\left(t, t^{\prime}\right)+I_{2,2}^{\varepsilon, v^{\varepsilon}}\left(t, t^{\prime}\right) .
\end{aligned}
$$

By (1.12) in [13], the Hölder inequality and the estimation (3.9) there exists $1 \leq \rho \leq p$ and $\kappa \in[0,1]$ such that

$$
\begin{aligned}
\mathbf{E}\left(\left\|I_{2,2}^{\varepsilon, v^{\varepsilon}}\left(t, t^{\prime}\right)\right\|_{p}^{q}\right) & \leq c \mathbf{E}\left(\int_{0}^{t^{\prime}-t}\left(t^{\prime}-t-s\right)^{-\frac{1}{2}+\frac{d}{4}(\kappa-1)}\left\|f\left(u^{\varepsilon, v^{\varepsilon}}(t+s, \cdot)\right)\right\|_{\rho} d s\right)^{q} \\
& \leq c\left|t^{\prime}-t\right|^{q\left(\frac{1}{2}+\frac{d}{4}(\kappa-1)\right)} \int_{0}^{t^{\prime}-t}\left(t^{\prime}-t-s\right)^{-\frac{1}{2}+\frac{d}{4}(\kappa-1)} \mathbf{E}\left(\left\|f\left(u^{\varepsilon, v^{\varepsilon}}(t+s, \cdot)\right)\right\|_{\rho}^{q}\right) d s \\
& \leq c\left|t^{\prime}-t\right|^{(q+1)\left(\frac{1}{2}+\frac{d}{4}(\kappa-1)\right)} .
\end{aligned}
$$

Using (3.14) in [4], Hölder inequality and the estimation (3.9), there exist $\theta \in] 0, \frac{1}{2}+\frac{d}{4}(\kappa-1)[$ and $\gamma \in$ ]$\frac{1}{\frac{1}{2}+\frac{d}{4}(\kappa-1)-\theta}, q[$ such that

$$
\begin{aligned}
\mathbf{E}\left(\left\|I_{2,1}^{\varepsilon, v^{\varepsilon}}\left(t, t^{\prime}\right)\right\|_{p}^{q}\right) & \leq c\left|t^{\prime}-t\right|^{\theta q} \mathbf{E}\left(\left\|f\left(u^{\varepsilon, v^{\varepsilon}}(\cdot, *)\right)\right\|_{L^{\gamma}\left([0, T], L^{\rho}(D)\right)}^{q}\right) \\
& \leq c\left|t^{\prime}-t\right|^{\theta q} .
\end{aligned}
$$

Concerning $I_{3}^{\varepsilon, v^{\varepsilon}}(t)$ we have

$$
\begin{aligned}
I_{3}^{\varepsilon, v^{\varepsilon}}\left(t^{\prime}\right)-I_{3}^{\varepsilon, v^{\varepsilon}}(t)= & \int_{0}^{t} \int_{D}\left[G_{t^{\prime}-s}(\cdot, y)-G_{t-s}(\cdot, y)\right] \sigma\left(u^{\varepsilon, v^{\varepsilon}}(s, y)\right) v^{\varepsilon}(s, y) d s d y \\
& +\int_{t}^{t^{\prime}} \int_{D} G_{t^{\prime}-s}(\cdot, y) \sigma\left(u^{\varepsilon, v^{\varepsilon}}(s, y)\right) v^{\varepsilon}(s, y) d s d y \\
\equiv & I_{3,1}^{\varepsilon, v^{\varepsilon}}\left(t, t^{\prime}\right)+I_{3,2}^{\varepsilon, v^{\varepsilon}}\left(t, t^{\prime}\right) .
\end{aligned}
$$

By Cauchy-Schwarz inequality, the fact that $\left\|v^{\varepsilon}\right\|_{\mathcal{H}_{T}} \leq N$ a.s. and by Lemma 1.8. in [13] we obtain the existence of $\eta>0$ such that

$$
\mathbf{E}\left(\left\|I_{3, i}^{\varepsilon, v^{\varepsilon}}\left(t, t^{\prime}\right)\right\|_{p}^{q}\right) \leq c\left|t^{\prime}-t\right|^{\eta q},
$$

for $i=1,2$.

Therefore, by (4.24)-(4.28) we obtain (4.17) for the first term. And arguing similarly and using the estimation (3.14) we obtain (4.17) for the second term. Hence, the condition (A2) is checked. 
Concerning (A1), it will be a consequence of the continuity of the mapping $h: \mathcal{H}_{T}^{N} \longrightarrow \mathcal{E}^{\alpha}$ with respect to the weak topology. It consists to consider $v,\left(v_{n}\right) \subset \mathcal{H}_{T}^{N}$ such that for any $g \in \mathcal{H}_{T}^{N}$,

$$
\lim _{n \longrightarrow+\infty}\left\langle v-v_{n}, g\right\rangle_{\mathcal{H}_{T}^{N}}=0,
$$

and to prove

$$
\lim _{n \longrightarrow+\infty}\left\|u^{v_{n}}-u^{v}\right\|_{\alpha, p}=0 .
$$

The proof will be omitted since we can proceed as for $(A 2)$ and by using the following estimate

$$
\sup _{\|v\| \leq N} \sup _{t \in[0, T]}\left\|u^{v}(t)\right\|_{p}<\infty
$$

which follows from Lemma 3.1. in [32].

Finally, since the conditions $(A 1)$ and $(A 2)$ are held, the proof of Theorem 4.1 is completed.

\section{Appendix}

We recall here some useful results that we have used in the proofs of our result. The following lemma gives well-known estimates on space and time increments for the Green function $G$. For the proof, we refer to [13] .

Lemma 5.1 There exists positive constants $c, \gamma$ and $\gamma^{\prime}$ satisfying $\gamma<4-d, \gamma \leq 2$ and $\gamma^{\prime}<1-\frac{d}{4}$ such that for all $y, z \in \mathcal{D}, 0 \leq s<t \leq T$ and $0 \leq h \leq t$ we have

1.

$$
\int_{0}^{t} \int_{\mathcal{D}}\left|G_{r}(x, y)-G_{r}(x, z)\right|^{2} d x d r \leq c|y-z|^{\gamma}
$$

2.

$$
\int_{0}^{t} \int_{\mathcal{D}}\left|G_{r+h}(x, y)-G_{r}(x, y)\right|^{2} d x d r \leq c|h|^{\gamma^{\prime}}
$$

3.

$$
\int_{s}^{t} \int_{\mathcal{D}}\left|G_{r}(x, y)\right|^{2} d x d r \leq c|t-s|^{\gamma^{\prime}}
$$

The following lemma is a version of the Garsia-Rademich-Rumsay lemma. For the proof, we refer to [14] and references therein.

Lemma 5.2 Let $\alpha] 0 ; 1]$ and $1<p \leq q$. Consider a sequence of stochastic processes $\left(Y_{n}\right)_{n}$ which belong to $C^{\alpha}\left([0, T] ; L^{p}(\mathcal{D})\right)$, and a sequence of stopping times $\left(\tau_{n}\right)$ such that

1. for any $t \in[0, T]$,

$$
\lim _{n \longrightarrow+\infty} \mathbf{E}\left(1_{\left\{t \leq \tau_{n}\right\}}\left\|Y_{n}(t, \cdot)\right\|_{p}^{q}\right)=0 ;
$$

2. there exists $\gamma>0$ such that for any $\left(t, t^{\prime}\right) \in[0, T]$

$$
\sup _{n} \mathbf{E}\left(1_{\left\{t \vee t^{\prime} \leq \tau_{n}\right\}}\left\|Y_{n}(t, \cdot)-Y_{n}\left(t^{\prime}, \cdot\right)\right\|_{p}^{q}\right) \leq c\left|t-t^{\prime}\right|^{\gamma+d},
$$

then, for any $1 \leq r<q$ and any $\theta<\frac{\gamma}{q}$ one has

$$
\lim _{n \longrightarrow+\infty} \mathbf{E}\left(1_{\left\{t \leq \tau_{n}\right\}}\left\|Y_{n}(t, \cdot)\right\|_{\theta, p, \tau_{n}}^{r}\right)=0
$$

where, for a stopping time $\tau$

$$
\|u\|_{\theta, p, \tau}:=\sup _{t \in[0, T \wedge \tau]}\|u(t)\|_{p}+\sup _{t \neq t^{\prime} t, t^{\prime} \in[0, T \wedge \tau]} \frac{\left\|u(t)-u\left(t^{\prime}\right)\right\|_{p}}{\left|t-t^{\prime}\right|^{\theta}} .
$$




\section{References}

[1] D. Antonopoulou, G. Karali, A. Millet. Existence and regularity of solution for a stochastic CahnHilliard/Allen-Cahn equation with unbounded noise diffusion. J. Diff. Equ. 15, 2383-2417, 2016.

[2] R. Azencott. Grandes déviations et applications. École d'Été de Probabilités de Saint-Flour VII, Lecture Notes in Math. Springer, New York, 774, 1176, 1980.

[3] P. Baldi and M. Cheleyat-Maurel. An extension of ventsel-freidlin estimates. In H. Korezlioglu and A. S. Üstünel, editors, Stochastic Analysis and Related Topics. Proceedings of a Workshop held in Silivri, Turkey, July 7-9, 1986, volume 1316 of Lecture Notes in Math., 305-327. Springer, 1988.

[4] L. Bo, Y. Jiang, Y. Wang. Stochastic Cahn-Hilliard equation with fractional noise. Stochastics and Dynamics, 8, (4), 643-665, 2008.

[5] L. Bo, K. Shi, Y. Wang. Support theorem for a stochastic Cahn-Hilliard equation. E. J. P., 15, (17), 484-525, 2010.

[6] M. Boué, P. Dupuis. A variational representation for certain functionals of Brownian motion. Ann. Probab. 26, 4, pp. 1641-1659, 1998.

[7] W. Bryc. Large deviations by the asymptotic value method. Diffusion Processes and Related Problems in Analysis, ed. M. Pinsky, vol. 1. Boston MA: Birkhauser, 447-472, 1990.

[8] A. Budhiraja, J. Chen, P. Dupuis. Large deviations for stochastic partial differential equations driven by a Poisson random measure. Stochastic Processes and their Applications, Volume 123, Issue 2, 523-560, 2013.

[9] A. Budhiraja, P. Dupuis. A variational representation for positive functionals of infinite dimensional Brownian motion. Probab. Math. Statist. 20, 39-61, 2000.

[10] A. Budhiraja, P. Dupuis, V. Maroulas. Large deviations for infinite dimensional stochastic dynamical systems. The Annals of Probability, 36, 4, 1390-1420, 2008.

[11] J. W. Cahn and J. E. Hilliard. Free energy of a nonuniform system. I. interfacial free energy. Journal of Chemical Physics, 28, 258, February 1958.

[12] C. Cardon-Weber. Large deviations for a Burgers'-type SPDE. Stochastic Processes and their Applications, 84, (1), 53-70, 1999.

[13] C. Cardon-Weber. Cahn-Hilliard stochastic equation: existence of the solution and of its density. Bernoulli, 7, (5), 777-816, 2001.

[14] C. Cardon-Weber, A. Millet. A support theorem for a generalized Burgers' SPDE. Potential Analysis, 15, (4), 361-408, 2001.

[15] F. Chenal, A. Millet.Uniform large deviations for parabolic SPDE's and applications. Stochastic Processes and their Applications, 72, 161-186, 1997.

[16] R. C. Dalang, Extending the martingale measure stochastic integral with applications to spatially homogeneous SPDE's Electronic J. Probab. 4, 1-29, 1999.

[17] A. Debussche and L. Dettori. On the Cahn-Hilliard equation with a logarithmic free energy. Nonlinear Anal., 24, (10), 1491-1514, 1995.

[18] A. Dembo, O. Zeitouni. Large deviations techniques and applications. Second edition. Applications of Mathematics (New York), 38. Springer-Verlag, New York, 1998. 
[19] J. Duan, A. Millet.Large deviations for the Boussinesq equations under random influences. Stoch. Proc. and Appl. 119, 6, 2052-2081, ,2009.

[20] C. M. Elliott. The Cahn-Hilliard model for the kinetics of phase separation. Mathematical models for phase change problems; vol 88 of the series International Series of Numerical Mathematics, pages 35-73, 1989 Birkhäser Verlag Basel.

[21] P. Dupuis, R. S. Ellis. A weak convergence approach to the theory of large deviations. Wiley, 1997.

[22] T. El Mellali, and M. Mellouk. Large deviations for a stochastic heat equation in spatial dimension $\mathbb{R}^{d}$ driven by a spatially correlated noise. Stochastic and Dynamics. 16, (1), 2016.

[23] M. I. Freidlin, A. D. Wentzell. Random Perturbations of Dynamical Systems. Springer-Verlag, New York, 1984.

[24] M. I. Freidlin, A. D. Wentzell. Reaction-diffusion equation with randomly perturbed boundary condition, Ann. Probab. 20 (2), 963-986, 1992.

[25] Y. Jiang, K. Shi, S. Wang. Stochastic Cahn-Hilliard equations driven by Poisson random measures. Sciences China Mathematics. 57 (12), 2563-2576, 2014.

[26] J. S. Langer. Theory of spinodal decomposition in alloys. Annals of Physics, 65:53-86, 1971.

[27] D. Marquez-Carreras, and M. Sarra. Large Deviation Principle for a Stochastic Heat Equation With Spatially Correlated Noise EJP, 8, 1-39, 2003.

[28] A. Novick-Cohen and L. A. Segel. Nonlinear aspects of the Cahn-Hilliard equation. Phys. D, 10, (3), 277-298, 1984.

[29] V. Ortiz-López, Marta Sanz-Solé. A Laplace Principle for a Stochastic Wave Equation in Spatial Dimension Three. Stochastic Analysis, 31-49. Springer, Berlin, Heidelberg, 2010.

[30] P. Priouret. Remarques sur les petites perturbations de systèmes dynamiques. Séminaire de probabilités XVI. Université de Strasbourg. Lecture notes Math, 920, 184-200, 1982.

[31] L. Setayechgar. Large deviations for a stochastic Burgers equation. Communications on Stochastic Analysis. 8 (2), 141-154, 2014.

[32] K. H. Shi, D. Tang, Y. J. Wang. Large Deviations for Stochastic Cahn-Hilliard Partial Differential Equations. Acta mathematica Sinica, English Series. 25, (7), 1157-1174, 2009.

[33] R. Sowers. Large deviations for a reaction diffusion equation with non- Gaussian perturbation. Ann. Probab. 20, 504-537, 1992.

[34] S. S. Sritharan, P. Sundar. Large deviations for the two-dimensional Navier-Stokes equations with multiplicative noise. Stoch. Proc. and Appl. 116, 1636-1659, 2006.

[35] S. R. S. Varadhan. Asymptotic probabilities and differential equations, Comm. Pure Appl. Math. 19, 261286, 1966.

[36] J.B. Walsh. An introduction to stochastic partial differential equations. In: Hennequin, P. L. (ed.) École d'été de probabilités de Saint-Flour XIV - 1984, Lect. Notes Math. vol. 1180, pp. 265-437. Springer, Berlin (1986). 
Département de Mathématiques

Centre Régional des Métiers de l'Éducation et de la Formation 80000 AGADIR

MAROC

e-mail: 1.boulanba@gmail.com,

MAP5, CNRS UMR 8145

Université Paris Descartes

45, RUE DES SAINTS-PÈreS

75270 Paris CedeX 6

FRANCE

e-mail: mohamed.mellouk@parisdescartes.fr 